\title{
Prognosis of treated hypertension
}

\author{
A. Breckenridge \\ From the Department of Clinical Pharmacology, Royal Postgraduate Medical School, \\ Hammersmith Hospital, London W.I2
}

The prognosis of patients with untreated severe hypertension is poor (Bechgaard, 1946). There has been a growing awareness in recent years that even patients with mild hypertension do not live as long as their normotensive counterparts, and as a result hypotensive therapy is now being offered to an increasing number of such patients. There has also been an improvement in the drugs used to lower blood pressure, so that recumbent as well as standing blood pressure can be reduced more easily and the side effects of the drugs used are not so noticeable.

We have recently examined the prognosis of all patients attending Hammersmith Hospital hypertension clinic during the years 1952 to 1967 (Breckenridge, Dollery, and Parry, 1970). In those 16 years, 1294 patients were seen and treated. One hundred and sixty-two patients presented with grade 4 (malignant) hypertension, 226 with grade 3 retinal changes, and 906 with less severe grades of hypertension, as judged by retinal changes. There has been an interesting change in the pattern of new patients being referred to the clinic over the years. Malignant hypertension has become progressively rarer, so that only 2 such patients were referred to our clinic in I966 and 1967. A peak was reached in 1960, when 17 new patients with malignant hypertension were seen. Similarly, the number of new patients with grade 3 retinopathy, 29 in 1962, fell to 8 in 1966, and 12 in 1967.

The sixteen years $1952-67$ were divided into two eight-year periods: 1952-59, when ganglion-blocking drugs formed the main type of treatment available, and 1960-67, when diuretics and adrenergic neurone-blocking drugs became available. For those patients with grade 4 (malignant) hypertension beginning treatment in the first eight-year period, the five-year survival was 25 per cent, and for the second period 34 per cent. In both eight-year periods the survival was better for female than for male patients. The most important single factor determining the outlook of these patients was the level of blood urea when the patients were originally seen. In both eight-year periods the prognosis was significantly better for patients with a blood urea below $40 \mathrm{mg} . / 100 \mathrm{ml}$. These figures reflect the same pattern as seen in other clinics: for example, Hodge, McQueen, and Smirk (I96I) describe a five-year survival of 38 per cent in patients seen in the years 1950-57 and note that the state of the patients' renal function was the most important single factor deciding their prognosis. The prognosis for patients with grade 3 retinopathy was significantly better than for patients with malignant hypertension. The five-year survival in the two eight-year periods was 70 per cent and 71 per cent, respectively. As in patients with malignant hypertension, the prognosis of female patients was better than that of men, and the initial level of the blood urea was an important factor in deciding their outlook. Untreated, the prognosis of patients with grade 3 retinopathy is very poor. Keith, Wagener, and Barker (1939) found that only 3 of 37 such patients were alive 5 years later. Thus treatment has afforded a considerable improvement in this group. We grouped the remaining patients in one category (called non-exudative retinopathy) and in them the five-year survival was 85 per cent for those starting treatment in the period 1952-59, and 87 per cent for those starting treatment in 1960-67.

When the survival of these patients is considered according to the level of their initial blood pressure several interesting facts emerge. Firstly, for male and female patients the prognosis at all levels of pressure is better in the second eight-year period than the first. Secondly, it is only above a pretreatment diastolic blood pressure of $140 \mathrm{~mm}$. $\mathrm{Hg}$ that there is a sharp decline in the survival of treated patients. This suggests that it may be the level of the blood pressure achieved by treatment which determines survival. Many patients with a pretreatment diastolic blood pressure above $140 \mathrm{~mm} . \mathrm{Hg}$ already have renal impairment before treatment is begun, and this is an important factor in determining prognosis. 
In these 16 years 290 patients died. In the years $1952-59$ there were 87 deaths, and in the years 1960-67, 203. Renal failure was the commonest cause of death in both periods, accounting for 44 per cent of the deaths in the first eight years and 29 per cent in the second. Cerebrovascular disease was the cause of death in 24 patients $(28 \%$ of the total deaths) in 1952-59, and 42 patients (21\%) in 1960-67. A striking change in the mortality due to ischaemic heart disease has been found. In the first eight-year period it accounted for 6 deaths ( $7 \%$ of the total), while in the second period 54 deaths $(27 \%$ of the total) were due to this cause. There was no evidence that blood pressure control was different in patients dying from these causes. Bauer (1966) has noted the same phenomenon in a sequence of 100 patients seen in 1955 and I00 in 1960, when myocardial infarction was the commonest cause of death in the second series.

These three causes of death, renal failure, cerebrovascular disease, and myocardial infarction, have been analysed in terms of length of time from diagnosis of hypertension until death. Of 97 patients dying from renal failure, $5 \mathrm{I}$ per cent died in the first year. This incidence decreased to 16 per cent in the second and 6 per cent in the third. The proportion of the 66 patients dying from stroke in the first year of treatment was 26 per cent, and in successive years the percentage was $21,17,11$, and 4 . The pattern for deaths from myocardial infarction was quite different. Only 6 per cent of these deaths occurred in the first year of followup, 22 per cent in the second, I I per cent in the third, and Io per cent in fourth and fifth years. From the fifth year of follow-up onwards myocardial infarction was the commonest cause of death.

Thus the pattern of hypertension seen has changed over the years. Phenomena similar to those studied in the United Kingdom have been documented from New Zealand, Australia, and the United States. The most important problem which must be solved to further improve the prognosis of patients with hypertension appears to be ischaemic heart disease, frequently occurring in patients whose blood pressure elevation is considered trivial. Attention to risk factors such as obesity, cigarette smoking, glucose tolerance, and lack of exercise may help with this problem. Numerically, malignant hypertension has become less important, and this may be because of earlier recognition and treatment of the disease.

\section{References}

Bauer, G. E. (1966). The changing pattern of hypertensive disease. Medical fournal of Australia, , , 698.

Bechgaard, P. (1946). Arterial hypertension. A followup study of one thousand hypertonics. Acta Medica Scandinavica, 172, Suppl. 3 .

Breckenridge, A., Dollery, C. T., and Parry, E. H. O. (1970). Prognosis of treated hypertension: changes in life expectancy and causes of death between I952 and 1967. Quarterly Fournal of Medicine, 39, $41 \mathrm{I}$.

Hodge, J. V., McQueen, E. G., and Smirk, H. (196I). Results of hypertensive therapy in arterial hypertension. British Medical fournal, $\mathbf{r}, \mathbf{1}$.

Keith, N. M., Wagener, H. P., and Barker, N. W. (1939). Some different types of essential hypertension: their course and prognosis. American Fournal of the Medical Sciences, 197, 332. 